EPJ manuscript No.

(will be inserted by the editor)

\title{
Stochastic resonance in finite arrays of bistable elements with local coupling
}

\author{
Manuel Morillo ${ }^{1}$, José Gómez Ordóñez ${ }^{1}$, José M. Casado ${ }^{1}$, Jesús Casado-Pascual ${ }^{1}$ and David \\ Cubero $^{2}$ a \\ 1 Física Teórica. Facultad de Física. Universidad de Sevilla. Apartado de Correos 1065. Sevilla 41080. \\ Spain \\ 2 Departamento de Física Aplicada I, EUP, Universidad de Sevilla, Calle Virgen de África 7, 41011 \\ Sevilla, Spain
}

\begin{abstract}
In this article, we investigate the stochastic resonance (SR) effect on a finite array of noisy bistable systems with nearest-neighbor coupling driven by a weak time-periodic driving force. The array is characterized by a collective variable. By means of numerical simulations, the signal-to-noise ratio (SNR) and the gain are estimated as functions of the noise and the interaction coupling strength. A strong enhancement of the SR phenomenon for this collective variable in comparison with SR in single unit bistable systems is observed. Gains larger than unity are obtained for some parameter values, indicating that the system is operating in a non-linear regime, albeit the smallness of the driving amplitude. The large values of the SNR observed are basically due to the fact that the output fluctuations are small and short lived, in comparison with their typical values in a linear regime. A non-monotonic behavior of the SNR with the coupling strength is also obtained.
\end{abstract}

\section{Introduction}

The study of the response of complex systems formed by coupled nonlinear noisy elements subject to the action of a time-periodic force brings into the picture a new ingredient with respect to the response of a single unit: the effect of the interactions. The interplay of noise, intrinsic nonlinear dynamics, driving forces and interactions render the response a very rich stochastic process. Certain aspects of the response are associated with what is called stochastic resonance (SR) [1,2], a phenomenon of interest to scientific fields other than Physics (as discussed, for instance, in [3]). SR is characterized by the non-monotonic behavior of some quantifiers with the noise strength. An enhancement of SR effects with respect to those observed in single units have been reported in several papers [4-8]. More recently [9-11], we have analyzed the collective response of a finite set of globally coupled bistable systems. We demonstrated that the response of the collective variable shows SR effects which are indeed much enhanced with respect to those in single bistable units. Actually, in those arrays, SR gains larger than unity were observed, we believe for the first time, for subthreshold sinusoidal driving forces. Those findings indicated that the arrays were indeed operating in nonlinear regimes. Similar results have been reported for single bistable units driven by a suprathreshold sinusoidal force [12], by subthreshold multifrequency forces [13-18], or by a subthreshold sinusoidal force in the presence of a strong, high-frequency monochromatic signal [19]. Nevertheless, to the best of our

\footnotetext{
a We acknowledge the support of the Dirección General de Enseñanza Superior of Spain (BFM200502884) and the Junta de Andalucía.
} 
knowledge, gains larger than unity have never been observed when the single bistable unit is driven by just a subthreshold sinusoidal force.

In a later work [20], the response of the collective variable of a finite array of globally coupled units to a rather weak driving force was analyzed. Two aspects of the response were studied: SR and noise induced phase frequency synchronization. The fact that SR effects were greatly amplified and that very good phase frequency synchronization was found in the response to a weak driver led us to indicate that the arrays were indeed operating in nonlinear regimes.

In this work, we extend the results of the previous work to other topologies of the array. In [8], arrays of nonlinear bistable units with nearest neighbor coupling are considered. In that work the response of a single oscillator is found to be enhanced due to the coupling to the other units in the chain. In [21], the authors consider an array of driven spins with Glauber dynamics and nearest neighbor interaction. They study not only the response of a single spin, but also the behavior of the global magnetization of the array. Their analysis of the system response is based on the linear response approximation. In both papers [8,21], the coupling strength between the elements of the chain are suggested as suitable parameters besides the noise strength to efficiently perform desired operations. In this work, rather than dealing with a globally coupled network, we will consider as in [8] that the bistable units have nearest neighbor interactions. We will still be interested in finite arrays. While in [8] the authors focus on the response of a single oscillator, we will concentrate here as in $[9,21]$ on the behavior of a collective or global variable characterizing the entire array rather than on a single individual. By contrast with the range of parameters analyzed in [21], we will be dealing here with situations where a linear response approximation is not valid.

The rest of the paper is as follows. In Section 2, we introduce the model system, fix notation and indicate the relevant variables that we will use to quantify the SR effect. In Section 3, we present the main results of our numerical simulations. In Section 4 we comment on the main conclusions of our paper.

\section{The model system}

We consider a set of $N$ identical bistable elements characterized by the variables $x_{i}(t)(i=$ $1, \ldots, N)$ with nearest neighbor interactions. The dynamics is given by stochastic evolution equations (in dimensionless form) of the type

$$
\dot{x}_{i}(t)=x_{i}(t)-x_{i}^{3}(t)+\frac{\theta}{2}\left[x_{i-1}(t)+x_{i+1}(t)-2 x_{i}(t)\right]+\sqrt{2 D} \xi_{i}(t)+F(t),
$$

subject to the conditions $x_{N+1}(t)=x_{1}(t), x_{0}(t)=x_{N}(t)$. The external driving force is periodic in time with period $T$, i. e., $F(t)=F(t+T)$. The term $\xi_{i}(t)$ represents a white noise with zero average and $\left\langle\xi_{i}(t) \xi_{j}(s)\right\rangle=\delta_{i j} \delta(t-s)$. In the $N \rightarrow \infty$ limit, this model becomes the classical $\phi^{4}$ model analyzed in [22]. In the absence of driving, it has also been used to model the dynamics of voltage pulses along myelinated nerves [23]. In the context of the ratchet effect, a similar model has been studied in [24].

We define a collective variable $S(t)$ as

$$
S(t)=\frac{1}{N} \sum_{j=1}^{N} x_{j}(t) .
$$

We will concentrate on the SR effects associated with the collective variable, when the system size, $N$, is kept finite and the amplitude of the driving term is small. By small we mean that when the driving force acts on a single isolated unit, SR is well described by linear response theory.

We will use the signal-to-noise ratio (SNR) of the collective behavior as the quantifier of the SR effects. Its definition requires the evaluation of the one-time correlation function defined as

$$
L(\tau)=\frac{1}{T} \int_{0}^{T} d t\langle S(t) S(t+\tau)\rangle_{\infty} .
$$


The notation $\langle\ldots\rangle$ indicates an average over the noise realizations and the subindex $\infty$ indicates the long time limit of the noise average, i. e., its value after waiting for $t$ long enough for the transients to die out. As indicated in our previous work [9], we can write

$$
L(\tau)=L_{\mathrm{coh}}(\tau)+L_{\mathrm{incoh}}(\tau)
$$

where the coherent part, $L_{\mathrm{coh}}(\tau)$,

$$
L_{\mathrm{coh}}(\tau)=\frac{1}{T} \int_{0}^{T} d t\langle S(t)\rangle_{\infty}\langle S(t+\tau)\rangle_{\infty},
$$

is periodic in $\tau$ with the period of the driving force, while the incoherent part, $L_{\text {incoh }}(\tau)$ arising from the fluctuations of the output $S(t)$ around its average value, decays to zero as $\tau$ increases. The output SNR, $R_{\text {out }}$, is

$$
R_{\text {out }}=\lim _{\epsilon \rightarrow 0^{+}} \frac{\int_{\Omega-\epsilon}^{\Omega+\epsilon} d \omega \tilde{L}(\omega)}{\tilde{L}_{\mathrm{incoh}}(\Omega)}=\frac{\tilde{L}_{\mathrm{coh}}(\Omega)}{\tilde{L}_{\mathrm{incoh}}(\Omega)},
$$

where $\Omega=2 \pi / T$ is the fundamental frequency of the driving force $F(t), \tilde{L}_{\mathrm{coh}}(\Omega)$ is the corresponding Fourier coefficient in the Fourier series expansion of $L_{\mathrm{coh}}(\tau)$, and $\tilde{L}_{\text {incoh }}(\Omega)$ is the Fourier transform at frequency $\Omega$ of $L_{\text {incoh }}(\tau)$.

We will also discuss the SR gain, $G$, defined as $[9]$

$$
G=\frac{R_{\text {out }}}{R_{\text {inp }}}
$$

where $R_{\text {inp }}$ is the SNR of the random input process formed by the arithmetic mean of the individual noise terms $\xi_{i}(t)$ plus the deterministic driving force $F(t)$, namely, $F(t)+\xi(t)$ with $\xi(t)=N^{-1} \sum_{i=1}^{N} \xi_{i}(t)$. The gain can be seen as a dimensionless parameter that compares the output SNR to that of the input and, in this sense, it measures the quality of the output relative to the input.

\section{Results}

Following the numerical procedure detailed in our previous works $[15,20]$, we have estimated the coherent and incoherent part of the collective correlation function $L(\tau)$, by integrating the Langevin equations, Eq. (1) and averaging over several thousand noise realizations. With this information, we evaluate numerically the integrals defining the Fourier coefficients at the driving frequency and, using Eqs. (6) and (7), the collective SNR and gain for a wide range of parameter values. In all the cases discussed below, we have used a weak driving rectangular force given by

$$
F(t)=(-1)^{n(t)} A,
$$

where $n(t)=\lfloor 2 t / T\rfloor,\lfloor z\rfloor$ is the floor function of $z$, i.e., the greatest integer less than or equal to $z$. The force amplitude will be taken to be $A=0.1$, much smaller than the barrier height of an isolated bistable unit while the fundamental frequency will be $\Omega=0.01$.

As noticed in [9], in the case of noninteracting units $(\theta=0)$, the SNR of the collective output is $N$ times larger than that of a isolated unit driven by the same force. Nonetheless, as discussed in [9], the gain associated with the collective output is just the same as the one of a single, isolated, unit. Thus, for the weak forces that we are considering here, the collective gain does not exceed unity, in agreement with the predictions of the linear response theory $[15,25$, 26]. The introduction of interactions between the bistable units drastically changes this picture and the enhancement of the SNR leads to the possibility of observing gains larger than unity for the weak driving forces considered. These facts can be observed in Fig. 1. 


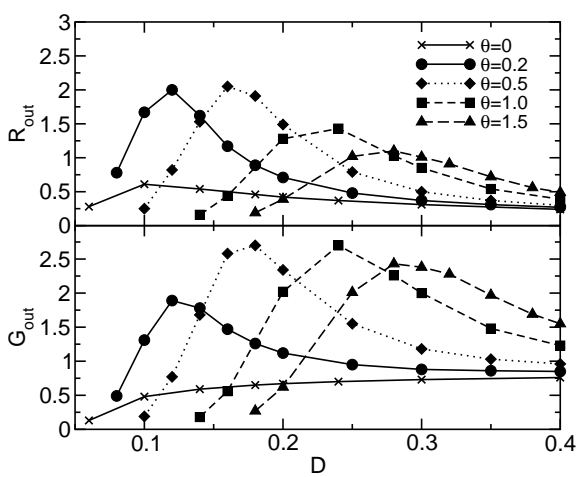

Fig. 1. The collective SNR, $R_{\text {out }}$, (upper panel) and the collective gain, $G$, (lower panel) vs. the noise strength $D$ for an array of $N=10$ bistable units driven by a rectangular driving force with amplitude $A=0.1$, fundamental frequency $\Omega=0.01$ and several values of the coupling parameter: $\theta=0$ (crosses), $n, 1, n, 1,1,1,5$ (triangles). Lines are a guide to the eye.

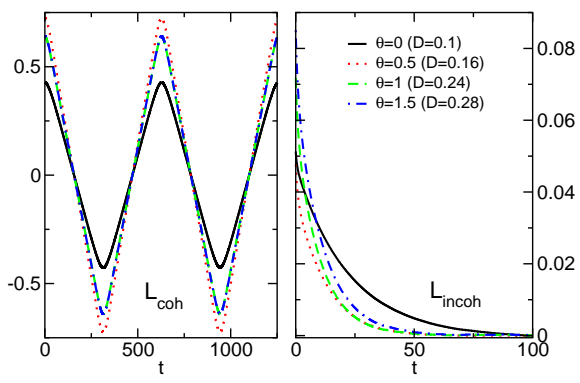

Fig. 2. The coherent part, $L_{\mathrm{coh}}(t)$, (left panel) and the incoherent part, $L_{\text {incoh }}(t)$, (right panel) of the correlation function of the collective variable for several values of the coupling parameter: $\theta=0$ (solid line), 0.5 (dotted), 1 (dashed), and 1.5 (dot-dashed), corresponding to the noise strength values $D=0.1,0.16,0.24$, and 0.28 , respectively. These noise values correspond to the peaks observed in $R_{\text {out }}$ in the upper panel of Fig. 1. Other parameter values: $N=10$, amplitude $A=0.1$ and fundamental frequency $\Omega=0.01$.

The non-monotonic behavior of the SNR for the collective variable with the noise strength observed in the upper panel of Fig. 1 is indicative of the SR phenomenon. In the upper panel of Fig. 1, we depict the behavior of the global $R_{\text {out }}$ for an array of $N=10$ identical particles with nearest neighbor coupling. The values of the interaction parameter range from small values $(\theta=0.2)$ to rather large ones $(\theta=1.5)$. The $R_{\text {out }}$ peak value depends on the coupling strength in such a way that as $\theta$ is increased, the noise value at which $R_{\text {out }}$ reaches its maximum is shifted slightly to higher values.

It is interesting to compare the time behavior of the coherent, $L_{\mathrm{coh}}(t)$, and incoherent, $L_{\text {incoh }}(t)$, parts of the collective correlation function. In the left panel of Fig. 2, we depict the time behavior of the coherent part for several values of the interaction strength $\theta$. For each value of $\theta$, the noise strength value is that at which the SNR is maximal. The periodicity of $L_{\text {coh }}(t)$ is clearly demonstrated. Its amplitude is only slightly dependent on the interaction strength. Its Fourier component at the fundamental driving frequency is precisely the numerator of $R_{\text {out }}$. Their peak values are not much different from those obtained in a single bistable unit driven by the same force at the same noise strength.

In the right panel of Fig. 2, the behaviors of the corresponding incoherent parts are depicted. It is remarkable the fast decay of the fluctuations as well as their small values. As the denominator of the SNR is the Fourier component of those decaying functions at the driving frequency, it is clear that those contributions are small. Consequently, the values of the SNR are expected to be much enhanced with respect to those values typical of SR in the linear regime. The enhancement of SR effects in arrays of interacting bistable units with respect to those in 


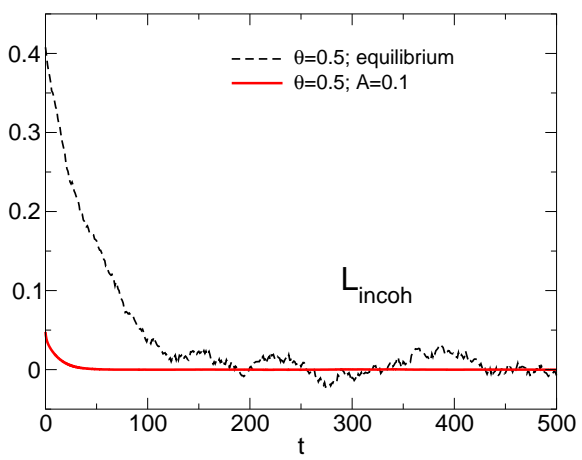

Fig. 3. Solid line: The incoherent part of the correlation function of the global variable for an array of $N=10$ bistable units driven by a rectangular driving force with amplitude $A=0.1$ and fundamental frequency $\Omega=0.01$. Dashed line: the equilibrium time correlation function of the global variable for the same array in the absence of driving. In both cases the coupling parameter is $\theta=0.5$ and the noise strength value $D=0.16$.

individual units is then basically a consequence of the strong reduction of the fluctuation level with respect to the one found in an single driven unit.

It should also be noted in the lower panel of Fig. 1 that the gain can be larger than unity for some ranges of noise strength values. This feature is a clear indication that the SR phenomenon observed in the array for the parameter values considered can not be described within the limits of a linear response theory. To further understand why linear response theory fails in the cases considered here, it seems useful to compare the behavior of the incoherent part of the one-time correlation function of the global variable and that of the equilibrium correlation function of the same global variable in an un-driven system. An example of such comparison is depicted in Fig. 3 for a coupling strength $\theta=0.5$. The graph clearly indicates that the equilibrium fluctuations are much larger and longer lasting than the fluctuations about the average behavior in the driven system. In the linear response theory description of SR, (see, for instance, [1] and references therein), it is assumed that the correlation function of the fluctuations around the average behavior in a driven system can be safely approximated by their corresponding equilibrium values in a un-driven one. This is clearly not the case for the the system at hand. A detailed study of the validity conditions of linear response theory can be found in $[27,28]$.

The peak values of $R_{\text {out }}$ also show a non-monotonic behavior with $\theta$ as depicted in Fig. 4. As $\theta$ is raised from zero up to 0.5 , there is an increase in the peak of the SNR value. This is due to the combination of two effects: i) the faster decay of the correlation function as $\theta$ is increased (see right panel in Fig. 2) with the subsequent decrease of the denominator in the SNR, and ii) the larger amplitude of the coherent part (see left panel in Fig. 2). On the other hand, as the $\theta$ values is further increased, the contribution of the coherent part decreases, while that of the incoherent part increases and, consequently, the SNR decreases as the coupling term increases. The gain also shows a non-monotonic behavior with $\theta$ as depicted in Fig. 4.

The complexity of the $N$-dimensional potential surface where the interacting particles move renders the task to give a simple explanation of the observed non-monotonic behavior with $\theta$ a difficult one. For an $N$-dimensional surface in the absence of interactions and driving forces, the whole surface is symmetrical about the origin with barriers of equal heights along each axis. The observed non-monotonic behavior can be rationalized in terms of the periodic rocking of the bistable potential independently along each axis. Each particle jumps over its corresponding barrier independently of the other particles, under the influence of the driving force and the noise. The central limit theorem can be safely used for independent subunits, so that the increase in the SNR values is just a size effect.

On the other hand, for coupled systems, the individual stochastic processes $x_{i}(t)$ are no longer independent and the central limit theorem alone is not enough to understand the reported results. The deformation of the potential surface due to the external driving and the interaction 


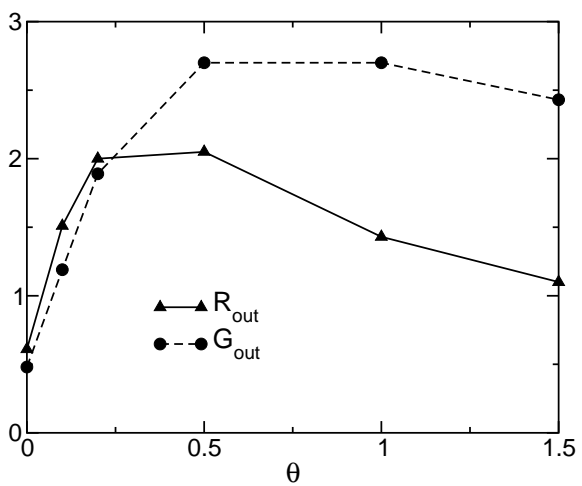

Fig. 4. The peak values of $R_{\text {out }}$ and $G$ vs. the coupling strength $\theta$ for $N=10, A=0.1$ and fundamental frequency $\Omega=0.01$.

term might very well reduce the height of the barriers, eliminate some of them and alter the location of the minima. Then, one can not rule out the possibility of the existence of new paths facilitating the transitions between the attractors. This being the case, a reduction of the fluctuation levels besides the one coming from the system size is to be expected. For a fixed small driving amplitude, the amount of distortion of the potential relief must depend on the strength of the coupling term $\theta$. For very small values of $\theta$, particles located beyond nearest neighbor positions are expected to be weakly correlated, with the correlation length increasing as $\theta$ increases. One might expect an increase on the SNR values as $\theta$ increases from zero, as the incoherent part of the correlation function basically decreases. This is due to the increasing easiness of transitions between attractors along the new paths. As $\theta$ is further increased, the distortion of the potential relief will increase, but at the same time, most of the particles along the chain will start to be strongly correlated. In other words, the motion of the collective variable will resemble more and more the motion of a particle on a single bistable potential. The chain will behave more and more like a rigid object. Jumps over the barriers become increasingly more difficult and with a decrease of the SNR values. There must be an intermediate interaction strength value so that a maximum SNR of the output is achieved.

It is interesting to study what happens in the case of a sinusoidal driving force with the same amplitude $A=0.1$ and frequency $\Omega=0.01$. In Fig. 5 we depict $R_{\text {out }}$ (lower panel) and $G$ (upper panel) vs. $D$ for several values of the coupling strength. As it can be seen, the SNR values are substantially smaller than the ones observed for a rectangular driving force (compare with Fig. 1). The gain is always below unity by contrast with the rectangular driving force, where the gain can reach values larger than 1.

In Fig. 6 we show the time dependence of the coherent and incoherent parts of the correlation function for an array of $N=10$ particles driven by the sinusoidal force. By comparison with Fig. 2 we see that the reason why the SNR values are much smaller for a sinusoidal driving than for a rectangular one is mainly that $L_{\text {incoh }}(t)$ is larger in the single frequency case than in the multi-frequency one. Concluding that because the gain in the case of sinusoidal driving is less than 1, a linear response theory description is adequate is not right. As shown in Fig. 7, $L_{\text {incoh }}(t)$ for a sinusoidal driving is too different from the equilibrium correlation function in an un-driven system.

A non-monotonic behavior of the SNR with the coupling constant $\theta$ for the sinusoidal driving also exists. The qualitative explanation given before for the rectangular driving still stands. Note, nonetheless, that the rocking of the multi-dimensional energy surface brought up by the sinusoidal driving is less drastic than the one produced by the rectangular one. Even though the forces have the same amplitude and fundamental frequency, the sinusoidal force introduces a bias in the energy relief with respect to that in the zero force case which is continuously changing with time. On the other hand, the rectangular signal keeps the surface biased most of the time, except during its instantaneous changes of value. The system has ample time to relax 

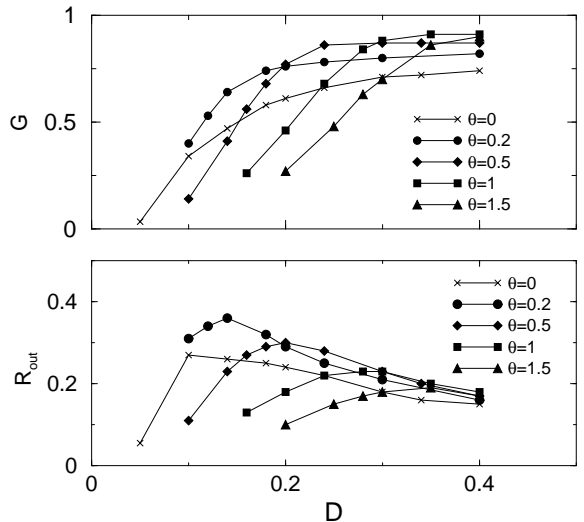

Fig. 5. The behavior of $R_{\text {out }}$ and $G$ with $D$ for several values of the coupling strength for an array of $N=10$, driven by a sinusoidal force with $A=0.1$ and frequency $\Omega=0.01$.

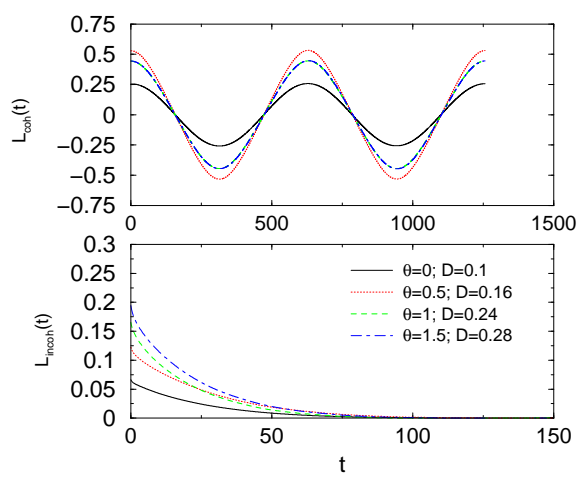

Fig. 6. The time behavior of $L_{\text {coh }}$ and $L_{\text {incoh }}$ for several values of the coupling strength for an array of $N=10$, driven by a sinusoidal force with $A=0.1$ and frequency $\Omega=0.01$.

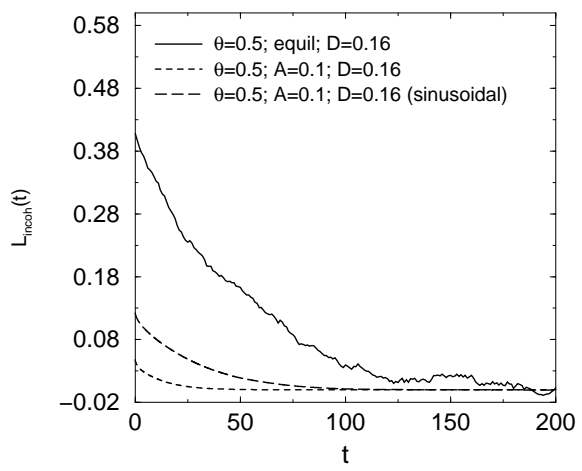

Fig. 7. Comparison of the time behavior of $L_{\text {incoh }}$ in an array of $N=10$ at equilibrium or driven by either a sinusoidal force with $A=0.1$ and frequency $\Omega=0.01$, or a rectangular one with the same amplitude and fundamental frequency.

during those biased intervals and this explains why the fluctuations are so drastically reduced in the case of a rectangular driving. 


\section{Conclusion}

In this work, we have explored the phenomenon of SR in finite arrays of noisy bistable units with nearest neighbor coupling, driven by time periodic forces of weak amplitude. Rather than analyzing the modification of a single unit behavior with respect to the one in the absence of any interaction, we have focused our attention in a collective variable describing the dynamics of the array as a whole.

A strong enhancement in the SR effects associated with the collective variable with respect to the one found in a system formed by a single unit has been demonstrated by means of numerical simulations. In particular, we have shown that, even for rather weak input amplitudes, the SNR has a non-monotonic behavior with the noise strength. The different values of the SNR quantifier are much larger than the corresponding ones found in a single unit system. Furthermore, the SR gain reaches values higher than unity for some values of the parameters, indicating that the array is operating in a nonlinear regime well beyond the limits of the regimes described by linear response theory. This is to some extent surprising as, for the driving amplitudes and frequencies considered, the response of a single unit system is well described by the linear response theory.

There are in principle two main reasons for the enhanced effects reported here. On the one hand, based on the central limit theorem, one can expect a decrease in the fluctuation levels with respect to those found in single unit systems simply because of the system size. On the other hand, as we have demonstrated in our numerical simulations, the size effect mechanism is not enough to explain the reported behavior. The presence of coupling terms between the subsystems is an essential ingredient to obtain gains larger than unity.

We believe that the main reason for the strong enhancement observed is due to the drastic reduction of the value of the incoherent correlation function and on its correlation time induced by the system size, the external driving and the coupling term between the different subunits. Indeed, a comparison with the decay of fluctuations in a finite system in the absence of driving with the same size and coupling parameter shows the relevance of the driving force.

An enhancement of the SR effects in finite size arrays with global coupling (mean field coupling) have also been reported by us. One of the goals of the present work is to demonstrate the robustness of our results, regardless of the topology of the connections between the different subunits. Indeed, a comparison of the numerical results reported here with those in [20] indicate that, at least for small size arrays $(N=10)$, the $R_{\text {out }}$ and gain values are not much different.

We have also noted that there exists a non-monotonic behavior of the SNR with respect to the coupling parameter $\theta$, besides the usual non-monotonic behavior with the noise strength. There are good reasons to believe that non-monotonic behaviors with respect to the system size do also exist. Indeed, system size resonances have been analyzed by other groups for systems of globally coupled nonlinear oscillators using cumulant expansion techniques [29] or nonequilibrium potentials [30]. Within a linear response theory description, they report system size resonance effects. We are presently investigating the issue of the dependence on the system size in coupled arrays (global and local coupling) for parameter regimes well beyond the linear response theory limits.

\section{References}

1. L. Gammaitoni, P. Hänggi, P. Jung and F. Marchesoni, Rev. Mod. Phys. 70, (1998) 223.

2. J. Casado-Pascual, J. Gómez-Ordóñez and M. Morillo, Chaos 15, (2005) 026115.

3. P. Hänggi, ChemPhysChem. 3, (2002) 285.

4. P. Jung, U. Behn, E. Pantazelou and F. Moss, Phys. Rev. A, 46, (1992) R1709.

5. A. Bulsara and G. Schmera, Phys. Rev. E, 47, (1993) 3734.

6. M. Morillo, J. Gómez-Ordóñez and J. M. Casado, Phys. Rev. E, 52, (1995) 316 ; J. M. Casado and M. Morillo, Phys. Rev. E, 52, (1995) 2088.

7. A. Neiman, L. Schimansky-Geier and F. Moss, Phys. Rev. E, 56, (1997) R9.

8. J. F. Lindner, B. K. Meadows, W. L. Ditto, M. E. Inchiosa and A. R. Bulsara, Phys. Rev. Lett 75, (1995) 3.

9. J. M. Casado, J. Gómez-Ordóñez and M. Morillo, Phys. Rev. E 73, (2006) 011109. 
10. D. Cubero, J. Casado-Pascual, J. Gómez-Ordóñez, J. Manuel Casado and M. Morillo, Phys. Rev.

E 75, (2007) 062102.

11. D. Cubero, Phys. Rev. E 77, (2008) 021112.

12. P. Hänggi, M. E. Inchiosa, D. Fogliatti and A. R. Bulsara, Phys. Rev. E 62, (2000) 6155.

13. K. Loerincz, Z. Gingl and L. B. Kiss, Phys. Lett. A, 224, (1996) 63.

14. Z. Gingl, P. Makra and R. Vajtai, Fluct. Noise Lett. 1, (2001) L181.

15. J. Casado-Pascual, C. Denk, J. Gómez-Ordóñez, M. Morillo and P. Hänggi, Phys. Rev. E, 68, (2003) 061104.

16. J. Casado-Pascual, J. Gómez-Ordóñez, M. Morillo and P. Hänggi, Phys. Rev. Lett. 91, (2003) 210601.

17. J. Casado-Pascual, J. Gómez-Ordóñez, M. Morillo and P. Hänggi, Phys. Rev. E 68, (2003) 061104.

18. J. Casado-Pascual, J. Gómez-Ordóñez and M. Morillo, Phys. Rev. E 69, (2004) 067101.

19. J. Casado-Pascual, D. Cubero and J. P. Baltanás, Europhys. Lett. 77, (2007) 50004.

20. M. Morillo, J. Gómez-Ordóñez and J. M. Casado, Phys. Rev. E 78, (2008) 021109.

21. L. Schimansky-Geier and U. Siewert, in Stochastic Dynamics, Lutz Schimansky-Geier; Thorsten Pöschel eds., Lecture Notes in Physics; 484 (Springer, Berlin 1997), 245.

22. F. Marchesoni, L. Gammaitoni and A. R. Bulsara, Phys. Rev. Lett. 76, (1996) 2609.

23. A. Scott, Neuroscience, A Mathematical Primer (Springer, New York, 2002). Chapter 7.

24. S. I. Denisov, E. S. Denisova and P. Hänggi, Phys. Rev. E 71, (2005) 016104.

25. M. I. Dykman, D. G. Luchinsky, R. Mannella, P. V. E. McClintock, N. D. Stein, and N. G. Stocks,

Il Nuovo Cimento 17D, (1995) 660.

26. M. DeWeese and W. Bialek, Il Nuovo Cimento 17D, (1995) 733.

27. J. Casado-Pascual, J. Gómez-Ordóñez, M. Morillo and P. Hänggi, Europhys. Lett. 58, (2002) 342.

28. J. Casado-Pascual, J. Gómez-Ordóñez, M. Morillo and P. Hänggi, Fluct. Noise Lett. 2, (2002) L127.

29. A. Pikovsky, A. Zaikin and M. A. de la Casa, Phys. Rev. Lett. 88, (2002) 050601.

30. B. von Haeften, G. Izús and H. S. Wio, Phys. Rev. E, 72, (2005) 021101. 